\title{
Networks of Mixed Canonical-Dissipative Systems and Dynamic Hebbian Learning
}

\author{
Julio Rodriguez ${ }^{2}$, Max-Olivier Hongler 1 \\ ${ }^{1}$ STI / IMT / LPMI, École Polytechnique Fédérale de Lausanne, \\ Lausanne, 1015, Switzerland \\ E-mail: julio.rodriguez@epfl.ch \\ ${ }^{2}$ Fakultät für Physik, Universität Bielefeld, \\ Bielefeld, 33501, Germany
}

Received: 30/09/08

Revised: 19/05/09

\begin{abstract}
We study the dynamics of a network consisting of $N$ diffusively coupled, stable-limit-cycle oscillators on which individual frequencies are parametrized by $\omega_{k}, k=1, \ldots, N$. We introduce a learning rule which influences the $\omega_{k}$ by driving the system towards a consensual oscillatory state in which all oscillators share a common frequency $\omega_{c}$. We are able to analytically calculate $\omega_{c}$. The network topology strongly affects the relaxation rate but not the ultimate consensual $\omega_{c}$. We report numerical simulations to show the learning mechanisms at work and confirm our theoretical assertions.
\end{abstract}

Keywords: mixed canonical-dissipative systems, diffusive coupling, Laplacian matrix, algebraic connectivity.

\section{Introduction}

In his famous monograph, ${ }^{1}$ I.I. Blekhman presents a general formulation of synchronization problems using the following dynamical system:

$$
\begin{aligned}
& \dot{x}_{k}=X_{k}\left(x_{k} ; \omega_{k}\right)+F_{k}\left(x_{1}, \ldots, x_{N}, u\right), \\
& \dot{u}_{k}=U\left(x_{1}, \ldots, x_{N}, u\right),
\end{aligned}
$$

where $X_{k}=\left(X_{k, 1}, \ldots, X_{k, n_{k}}\right), F_{k}=\left(F_{k, 1}, \ldots, F_{k, n_{k}}\right)$ and $U=\left(U_{1}, \ldots, U_{v}\right)$ are $n_{k}-$ and $v$-dimensional vector fields, respectively. The $\omega_{k}$ are sets of fixed parameters of $s_{k}$ elements. The $F_{k}$ and $U$ determine the connections between the local dynamics $X_{k}, k=1, \ldots, N$. The mathematical formulation in Eqs.(1) covers a broad class of interacting dynamical systems, ranging from coupled pendulums to complex networks. In this article, we generalize Eps.(1) by proposing the following system:

$$
\begin{aligned}
\dot{x}_{k} & =X_{k}\left(x_{k}, \omega_{k}\right)+F_{k}\left(x_{1}, \ldots, x_{N}, u\right) \\
\dot{u}_{k} & =U\left(x_{1}, \ldots, x_{N}, u\right) \\
\dot{\omega}_{k} & =\Omega_{k}\left(x_{1}, \ldots, x_{N}, \omega_{k}\right)
\end{aligned}
$$

where $\Omega_{k}=\left(\Omega_{k, 1}, \ldots, \Omega_{k, s_{k}}\right)$ is a $s_{k}$-dimensional vector field determining the dynamics of what was beforehand parameters, and what now becomes state variables $\omega_{k}$. Indeed, Eqs.(2) allow the parameters $\omega_{k}$ in Eqs.(1) to evolve in time according to $\Omega_{k}$, and thus to become variables of the system.

Our basic motivation originates from a recent paper $^{2}$ where L. Righetti et al. show how to im- 
plement what they call a Dynamic Hebbian Learning (DHL) process by perturbing nonlinear parametric oscillators with an external time-dependent signal. As a paradigmatic illustration, they consider an non-autonomous parametric Hopf oscillator (HO), defined in its phase space by the following system of equations:

$$
\begin{aligned}
\dot{x} & =+\omega y+\left(1-x^{2}-y^{2}\right) x+\varepsilon \sin (\Omega t), \\
\dot{y} & =-\omega x+\left(1-x^{2}-y^{2}\right) y, \\
\dot{\omega} & =\varepsilon \sin (\theta(t)) \sin (\Omega t),
\end{aligned}
$$

where $\varepsilon$ is a small positive constant, $\sin (\Omega t)$ externally perturbs the basic dynamics of the $\mathrm{HO}(\Omega$ is a positive constant), and where $\theta(t):=\arctan \left(\frac{y(t)}{x(t)}\right)$. This is a particular case of Eqs.(2) where the system is reduced to only one local dynamics (the Hopf oscillator), where the connection is given by the perturbing periodic signal, and where the third line of Eqs.(3) determines the dynamics on the frequency parameter $\omega$ which is now to be seen as a variable.

The DHL process manifests itself by the fact that the frequency of the underlying $\mathrm{HO} \omega$ asymptotically approximates $\Omega$, which is the frequency of the external input signal. In other words, the external signal "plastically" deforms the original limit cycle dynamics. We speak about plasticity to reflect the fact that, once this deformation is realized, it subsists even if the external input is removed. This generic behavior can be qualitatively understood by the fact that the external perturbing signal gradually affects the frequency $\omega$ on the limit cycle $\mathscr{L}$ (for Eqs.(3), $\mathscr{L}:=\left\{(x, y) \in \mathbb{R}^{2} \mid x^{2}+y^{2}=1\right\}$ ), but leaves the shape of $\mathscr{L}$ essentially invariant.

The main aim of the present paper is to substitute in Eqs.(3) the role played by the external signal by the dynamics delivered by other limit cycle oscillators, and to study the resulting mutual DHL process. More generally, we will consider a collection $\left\{\mathscr{O}_{k}\right\}_{k=1}^{N}$ of independent mixed canonicaldissipative systems (MCD), ${ }^{345}$ which exhibit limit cycles $\mathscr{L}_{k}$ and different individual $\omega_{k}, k=1,2, \ldots, N$ on $\mathscr{L}_{k}$. The action of the dissipative mechanism is to stabilize the orbits on $\mathscr{L}_{k}$, and the canonic part of the vector field (i.e. its Hamiltonian part) is responsible for the circulation on the limit cycles. In our class of models the mutual interactions between the $\mathscr{O}_{k}$ are characterized by:

a) a network $\mathscr{N}$ of diffusively coupled $\mathscr{O}_{k}$ - i.e. the row elements of the associated Laplacian coupling matrix of the network add up to zero.

b) a dynamic Hebbian learning mechanism (DHL). We allow the $\omega_{k}$ to behave as additional variables and implement couplings between these variables and the whole dynamics. Qualitatively speaking, the DHL coupling rule essentially affects the frequency on the limit cycles $\mathscr{L}_{k}$ while keeping the shape of $\mathscr{L}_{k}$ approximately unchanged.

The DHL process and the resulting "plasticity" of the dynamics confers a fundamentally different perspective compared to the abundantly studied synchronization networks of limit cycle oscillators. Indeed, interactions of the DHL type offer the possibility to drive the dynamics into a global (identical for all $\mathscr{O}_{k}$ ), stable oscillatory state which, once reached, remains "frozen" even when the interactions are removed. This final oscillatory behavior shared by all $\mathscr{O}_{k}$ will be called the consensual oscillatory state. In this context, a (non-exhaustive) list of issues to be addressed in this paper will read:

1) How to calculate the frequency $\omega_{c}$ characterizing the final consensual state ?

2) How does the consensual frequency depend on the Laplacian matrix associated to the network ?

3) How does the network influence the convergence rate towards the the final consensual state?

In section 2 we propose the construction of an analytically soluble class of coupled oscillators with mutual interactions leading to a DHL rule. A paradigmatic illustration of this class of dynamics is thoroughly studied in section 3, where explicit and fully analytical answers to questions 1) to 3 ) can be given. Future research perspectives and conclusion will be found in section 5 .

\section{Construction of a DHL network dynamics}

The collection $\left\{\mathscr{O}_{k}\right\}_{k=1}^{N}$ of oscillators will be chosen to belong to the class of mixed canonical-dissipative 
systems which we briefly expose in 2.1 .

\subsection{Mixed canonical-dissipative systems}

A member of our collection $\left\{\mathscr{O}_{k}\right\}_{k=1}^{N}$ will be defined as:

$$
\mathscr{O}_{k}\left\{\begin{array}{l}
\dot{x}_{k}=+\omega_{k} \frac{\partial H_{k}}{\partial y_{k}}+g_{k}\left(H_{k}\right) \frac{\partial H_{k}}{\partial x_{k}}, \\
\dot{y}_{k}=\underbrace{-\omega_{k} \frac{\partial H_{k}}{\partial x_{k}}}_{\text {conservative evolution }}+\underbrace{g_{k}\left(H_{k}\right) \frac{\partial H_{k}}{\partial y_{k}},}_{\text {dissipative evolution }}
\end{array}\right.
$$

where $H_{k}: \mathbb{R}^{2} \rightarrow \mathbb{R}^{+}$are evaluated at $\left(x_{k}, y_{k}\right)$ and $g_{k}$ : $\mathbb{R}^{+} \rightarrow \mathbb{R}, k=1, \ldots, N$. The $H_{k}$ functions are $C^{2}$ and positive definite and play the role of Hamiltonians (i.e. energy). Subsequently, we shall assume that $H_{k}\left(x_{k}, y_{k}\right)=\mathscr{E}_{k}$ uniquely defines a set of closed (concentric) curves $\mathscr{L}_{k}\left(\mathscr{E}_{k}\right)$ in $\mathbb{R}^{2}$ surrounding the origin. The $g_{k}$ functions are $C^{1}$, and $g_{k}\left(H_{k}\left(x_{k}, y_{k}\right)\right)$ are nonconservative terms which, according to the value of $H_{k}$, feed or dissipate energy from the Hamiltonian system. In particular, if $g_{k}\left(H_{k}\left(x_{k}, y_{k}\right)\right)$ vanishes for $H_{k}\left(x_{k}, y_{k}\right)=\mathscr{E}_{k}$, the dynamics is purely conservative (i.e. only the canonical part drives the dynamics) and we have:

$$
H_{k}\left(x_{k}, y_{k}\right)=\mathscr{E}_{k} \text { defines the limit cycle } \mathscr{L}_{k}\left(\mathscr{E}_{k}\right)
$$

with

$$
\mathscr{L}_{k}\left(\mathscr{E}_{k}\right):=\left\{(x, y) \in \mathbb{R}^{2} \mid H_{k}(x, y)=\mathscr{E}_{k}\right\} .
$$

The stability of the $\mathscr{L}_{k}\left(\mathscr{E}_{k}\right)$ will be determined by:

$$
\begin{aligned}
& g_{k}\left(H_{k}\right)>0 \text { in } \mathscr{A}_{k} \\
& g_{k}\left(H_{k}\right)<0 \text { in } \mathbb{R}^{2} \backslash \overline{\mathscr{A}_{k}} \quad \Rightarrow \quad \mathscr{L}_{k}\left(\mathscr{E}_{k}\right) \text { is stable, } \\
& g_{k}\left(H_{k}\right)<0 \text { in } \mathscr{A}_{k} \\
& g_{k}\left(H_{k}\right)>0 \text { in } \mathbb{R}^{2} \backslash \overline{\mathscr{A}_{k}} \quad \Rightarrow \quad \mathscr{L}_{k}\left(\mathscr{E}_{k}\right) \text { is unstable, }
\end{aligned}
$$

where $\mathscr{A}_{k}$ stands for the interior of $\mathscr{L}_{k}\left(\mathscr{E}_{k}\right)$, (i.e. $\left.\mathscr{A}_{k}:=\left\{(x, y) \in \mathbb{R}^{2} \mid H_{k}(x, y)<\mathscr{E}_{k}\right\}\right)$. Therefore, for $g_{k}\left(\mathscr{E}_{k}\right)=0$ and when $\mathscr{L}_{k}\left(\mathscr{E}_{k}\right)$ is stable, the energytype control $g_{k}\left(H_{k}\left(x_{k}, y_{k}\right)\right)$ drives all orbits towards the stable limit cycle $\mathscr{L}_{k}\left(\mathscr{E}_{k}\right)$, which is hence an attractor.
The system defined by Eqs.(4) belongs to the general class of mixed canonical-dissipative dynamics (MCD) (Ref. ${ }^{3},{ }^{4}$ and ${ }^{5}$ ). Subsequently, we shall use the short hand notation:

$$
\begin{array}{r}
P_{k}\left(x_{k}, y_{k}, \omega_{k}\right):=+\omega_{k} \frac{\partial H_{k}}{\partial y_{k}}+g_{k}\left(H_{k}\right) \frac{\partial H_{k}}{\partial x_{k}}, \\
Q_{k}\left(x_{k}, y_{k}, \omega_{k}\right):=-\omega_{k} \frac{\partial H_{k}}{\partial x_{k}}+g_{k}\left(H_{k}\right) \frac{\partial H_{k}}{\partial y_{k}},
\end{array}
$$

with evaluation at $\left(x_{k}, y_{k}\right)$. Observe that in Eqs.(4), we restrict ourselves to MCD for which $\omega_{k}$ are constant. Having defined the individual dynamics, we can characterize their interactions.

\subsection{Network of diffusively coupled oscillators}

The interactions between the MCD given by Eqs.(4) will be realized via a simply connected network $\mathscr{N}$ with $N$ vertices and without loops (i.e. its adjacent matrix $A$ is such that, for the $j^{\text {th }}$ edge, $A_{j, j}=0$ and $A_{i, j} \in\{0,1\}$ for $\left.j \neq i, j=1, \ldots, N\right)$. Let $L$ be the associated Laplacian matrix, namely $L:=A-D$, where $D$ is the diagonal matrix with $D_{j, j}$ being the degree of edge $j$. Accordingly, we now consider the dynamics:

$$
\begin{aligned}
& \dot{x}_{k}=P_{k}\left(x_{k}, y_{k}, \omega_{k}\right)+C_{\text {MCD }} C_{k} x \\
& \dot{y}_{k}=\underbrace{Q_{k}\left(x_{k}, y_{k}, \omega_{k}\right)}_{\text {diffusive coupling }}+\underbrace{C_{k} y}_{k}
\end{aligned}
$$

with $C_{k} x$ and $C_{k} y$ reading as:

$$
\begin{aligned}
& C_{k} x:=\varepsilon_{1}(x, y) \sum_{j=1}^{N} L_{k, j} x_{j}, \\
& C_{k} y:=\varepsilon_{2}(x, y) \sum_{j=1}^{N} L_{k, j} y_{j},
\end{aligned}
$$

where $0 \leqslant \varepsilon_{l}(x, y)<\varepsilon, l=1,2$ not simultaneously vanishing and $x:=\left(x_{1}, \ldots, x_{N}\right), y:=\left(y_{1}, \ldots, y_{N}\right)$.

Finally, we now introduce the DHL process into the dynamics. 


\subsection{Dynamic Hebbian learning for mixed canonical-dissipative systems}

Directly inspired by Eqs.(3) we now propose our generalized DHL in the context of Eqs.(6). For $k=1, \ldots, N$, the dynamical system is given by:

$$
\begin{aligned}
& \dot{x}_{k}=P_{k}\left(x_{k}, y_{k}, \omega_{k}\right)+C_{k} x, \\
& \dot{y}_{k}=Q_{k}\left(x_{k}, y_{k}, \omega_{k}\right)+C_{k} y, \\
& \dot{\omega}_{k}=\underbrace{\mathrm{K}_{k}\left[D y C_{k} x-D x C_{k} y\right],}_{\text {DHL mechanism }}
\end{aligned}
$$

with $D y$ and $D x$ reading as:

$$
\begin{aligned}
& D y:=\eta_{1}(x, y) \sum_{j=1}^{N} \frac{\partial H_{j}}{\partial y_{j}}, \\
& D x:=\eta_{2}(x, y) \sum_{j=1}^{N} \frac{\partial H_{j}}{\partial x_{j}} .
\end{aligned}
$$

where $0 \leqslant \mathrm{~K}_{k} \leqslant \kappa$ is a set of learning coupling strengths, and $0 \leqslant \eta_{l}(x, y) \leqslant \eta, l=1,2$ are not simultaneously vanishing.

Observe at this point that the dynamics defined by Eqs.(7) exhibits the salient features of the basic model given by Eqs.(3). We have:

- when $C_{k} x=C_{k} y=0$, and for appropriate choices of the $g_{k}\left(H_{k}\left(x_{k}, y_{k}\right)\right)$ terms, (see Eq.(5)), the local dynamics exhibits a stable limit cycle $\mathscr{L}_{k}$,

- on the limit cycle $\mathscr{L}_{k}$, the local dynamics obeys a (conservative) canonical Hamiltonian motion,

- a DHL-type mechanism explicitly affects the frequency $\omega_{k}$ of the orbits on $\mathscr{L}_{k}$.

For simplicity and without lost of generality, from now on we shall systematically make $\varepsilon_{1}(x, y)=$ $\varepsilon_{2}(x, y)=1$ and $\eta_{1}(x, y)=\eta_{2}(x, y)=1$ in Eqs.(7).

Proposition 1. Let $\mathrm{K}_{k}>0$ for all $k$ in the system defined by Eqs. (7). Then:

$$
J:=\sum_{k=1}^{N} \frac{\omega_{k}(t)}{\mathrm{K}_{k}}
$$

is a constant of the motion.

\section{Proof.}

$$
\begin{aligned}
\sum_{k=1}^{N} \frac{\dot{\omega}_{k}}{\mathrm{~K}_{k}} & =\sum_{k=1}^{N} D y C_{k} x-\sum_{k=1}^{N} D x C_{k} y \\
& =D y \sum_{k=1}^{N} \sum_{j=1}^{N} L_{k j} x_{j}-D x \sum_{k=1}^{N} \sum_{j=1}^{N} L_{k j} y_{j} \\
& =D y \sum_{j=1}^{N} x_{j} \sum_{k=1}^{N} L_{k j}-D x \sum_{j=1}^{N} y_{j} \sum_{k=1}^{N} L_{k j} \\
& =0 .
\end{aligned}
$$

where the last equality identically vanishes due to the diffusive character of the coupling matrix $L$.

Proposition 2. Assume that we have a collection of identical MCD systems (i.e. $H_{k} \equiv$ $H$ for all $k$ ) admitting, in absence of coupling, the same stable limit cycle $\mathscr{L}_{c}:=\mathscr{L}_{k}\left(\mathscr{E}_{c}\right)$ for all $k$ (i.e. for a fixed energy level $\mathscr{E}_{c}$ common to all oscillators, we suppose that $\left.g_{k}\left(\mathscr{E}_{c}\right)\right)=0$ for all $k$ ). Then, the synchronized orbit given by $\mathscr{S}(t):=\left(x_{s}(t), y_{s}(t), \omega_{c}, \ldots, x_{s}(t), y_{s}(t), \omega_{c}\right) \in \mathbb{R}^{3 N}$, with $\omega_{c}=$ constant and with:

$$
g_{k}\left(H\left(x_{s}(t), y_{s}(t)\right)\right)=g_{k}\left(\mathscr{E}_{c}\right)=0,
$$

is an exact solution of the dynamical system defined by Eqs.(7).

Proof. For the synchronized orbit, we have $x_{k}(t)=$ $x_{s}(t), y_{k}(t)=y_{s}(t)$ and $\omega_{k}(t)=\omega_{c}(t)$ for all $k$. The diffusive nature of the coupling implies that the terms $C_{k} x=C_{k} y=0$ and therefore $\dot{\omega}_{k}(t)=0$. Hence, the $\omega_{k}(t)$ are all the same constant $\omega_{c}$.

So far, we have introduced a globally nonconservative dynamical system given by Eqs.(7) for which an explicit orbit $\mathscr{S}(t)$ is known. In addition, our dynamics possesses one constant of motion $J$ given by Eq. (8). One may now question whether the orbit $\mathscr{S}(t)$ corresponds to a stable solution of the globally non-conservative dynamics. As usual, linearizing the dynamics around $\mathscr{S}(t)$ provides information regarding its stability - this will be explicitly performed in section 3 for systems with an underlying circular symmetry. To make headway, assume 
that $\mathscr{S}(t)$ is indeed a stable solution of the dynamics given by Eqs.(7) and that we have $\lim _{t \rightarrow \infty} \omega_{k}(t)=\omega_{c}$ for all $k$. Hence, $\omega_{c}$ corresponds to the consensual frequency on the common limit cycle $\mathscr{L}_{c}$. In this case, Proposition 1 and 2 provide explicit answers to questions 1) and 2) raised in the introduction. Indeed, Eq.(8) enables us to write:

if $\lim _{t \rightarrow \infty} \omega_{k}(t)=\omega_{c}$ then $\sum_{k=1}^{N} \frac{\omega_{c}}{\mathrm{~K}_{k}}=\sum_{k=1}^{N} \frac{\omega_{k}(0)}{\mathrm{K}_{k}}$

and therefore, we end with:

$$
\omega_{c}=\frac{\sum_{j=1}^{N} \frac{\omega_{j}(0)}{\mathrm{K}_{j}}}{\sum_{j=1}^{N} \frac{1}{\mathrm{~K}_{j}}} .
$$

From Eq.(10), we conclude that the consensual frequency $\omega_{c}$ depends on the distribution of initial conditions $\omega_{k}(0)$ and on the coupling strength $\mathrm{K}_{k}$ for $k=1, \ldots, N$, but it does not depend on the coupling matrix $L$ and therefore not on the topology of the coupling network. However, we shall see that $L$ directly affects the convergence rate towards the consensual orbit $\mathscr{S}(t)$.

\section{Network of coupled Hopf oscillators}

In this section, we focus on a situation where $H_{k} \equiv H$ for all $k$ and where the underlying Hamiltonian reads as $H(x, y)=H\left(x^{2}+y^{2}\right)=H\left(r^{2}\right)$. The circular symmetry implies that the consensual limit cycle $\mathscr{L}_{c}$ is a circle and the circulation is a uniform rotation with the consensual frequency given by Eq.(10). Due to the cylindrical symmetry, it is advantageous to express the dynamics in polar coordinates:

$$
\begin{aligned}
\dot{r}_{k} & =2\left(1-r_{k}^{2}\right) r_{k}+\sum_{j=1}^{N} L_{k, j} r_{j} \cos \left(\phi_{k}-\phi_{j}\right) \\
\dot{\phi}_{k} & =-2 \omega_{k}-\frac{1}{r_{k}}\left(\sum_{j=1}^{N} L_{k, j} r_{j} \sin \left(\phi_{k}-\phi_{j}\right)\right) \\
\dot{\omega}_{k} & =\mathrm{K}_{k}\left(\sum_{l=1}^{N}\left(\sum_{j=1}^{N} L_{k, j} r_{l} r_{j} \sin \left(\phi_{l}-\phi_{j}\right)\right)\right) .
\end{aligned}
$$

Note that in the non-parametric case (i.e. when $\left.\omega_{k}(t)=\omega_{k}\right)$, the phase dynamics in Eqs.(11) coincides with the Kuramoto model in the presence of a general coupling network as discussed in ${ }^{6}$. Here, the exact solution of Eqs. (11) on which perturbations will now be added, simply reads as:

$$
\begin{aligned}
\mathscr{S}_{p c}(t) & =\left(r_{s}(t), \theta_{s}(t), \omega(t), \ldots, r_{s}(t), \theta_{s}(t), \omega_{s}(t)\right) \\
& =\left(1,-2 \omega_{c} t, \omega_{c}, \ldots, 1,-2 \omega_{c} t, \omega_{c}\right) \in \mathbb{R}^{3 N} .
\end{aligned}
$$

Rearranging the variables in Eqs.(11) by using the permutation $3(k-1)+n \mapsto N(n-1)+k(k=$ $1, \ldots, N \quad n=1,2,3)$ and linearizing around $\mathscr{S}_{p c}(t)$ enables us to write:

$$
\left(\begin{array}{c}
\dot{\rho} \\
\dot{\delta} \\
\dot{\varepsilon}
\end{array}\right)=\left(\begin{array}{ccc}
L-4 \mathrm{Id} & \mathbb{O} & \mathbb{O} \\
\mathbb{O} & L & -2 \mathrm{Id} \\
\mathbb{O} & -2[K] L & \mathbb{O}
\end{array}\right)\left(\begin{array}{l}
\rho \\
\delta \\
\varepsilon
\end{array}\right)
$$

where Id is the identity matrix, $[K]$ is a diagonal matrix with $\mathrm{K}_{1}, \mathrm{~K}_{2}, \ldots, \mathrm{K}_{N}$ on the diagonal and where $\rho:=\left(\rho_{1}, \ldots, \rho_{N}\right), \delta:=\left(\delta_{1}, \ldots, \delta_{N}\right)$ and $\varepsilon:=$ $\left(\varepsilon_{1}, \ldots, \varepsilon_{N}\right)$ are perturbations. To fulfill the conservation law given by Eq.(8), we further impose the that:

$$
\sum_{j=1}^{N} \frac{\varepsilon_{j}(0)}{\mathrm{K}_{j}}=0, \quad\left(\text { here } \mathrm{K}_{k} \text { is constant for all } k\right) .
$$

To explicitly exhibit the influence of the network, we take a case where $\mathrm{K}_{k}:=\mathrm{K}$ for all $k$. Since $L$ is symmetric, there exists an orthogonal matrix $V$ such that $V^{\top} L V$ is a diagonal matrix $[\lambda]$ with its spectrum $\left\{\lambda_{k}\right\}_{k=1}^{N}$ on the diagonal. The network being connected, there exists a unique $j$ such that $\lambda_{j}$ is zero and the rest of the spectrum is strictly negative. Without lost of generality, we assume $\lambda_{1}=0$. Changing the basis of the system by means of a $(3 \times 3)$-bloc matrix with $V^{\top}$ on its diagonal gives us:

$\left(\begin{array}{c}\dot{\tilde{\rho}} \\ \dot{\widetilde{\delta}} \\ \dot{\tilde{\varepsilon}}\end{array}\right)=\left(\begin{array}{ccc}{[\lambda]-4 \mathrm{Id}} & \mathbb{O} & \mathbb{O} \\ \mathbb{O} & {[\lambda]} & -2 \mathrm{Id} \\ \mathbb{O} & -2 \mathrm{~K}[\lambda] & \mathbb{O}\end{array}\right)\left(\begin{array}{c}\widetilde{\rho} \\ \widetilde{\delta} \\ \widetilde{\varepsilon}\end{array}\right)$. 
The upper left $(N \times N)$-bloc in Eqs.(15) has $N$ real negative eigenvalues, and the rest of the system is described by the following $(2 \times 2)$-blocs:

$$
\left(\begin{array}{c}
\dot{\tilde{\delta}}_{k} \\
\dot{\widetilde{\varepsilon}}_{k}
\end{array}\right)=\left(\begin{array}{cc}
\lambda_{k} & -2 \\
-2 \mathrm{~K} \lambda_{k} & 0
\end{array}\right)\left(\begin{array}{c}
\widetilde{\delta}_{k} \\
\widetilde{\varepsilon}_{k}
\end{array}\right) .
$$

For $k=1$, we have:

$$
\dot{\widetilde{\delta}}_{1}=-2 \widetilde{\varepsilon}_{1} \quad \dot{\widetilde{\varepsilon}}_{1}=0
$$

This is a direct consequence of the conservation law Eq.(8) and the restriction imposed by Eq.(14). For $k \neq 1$ the eigenvalues of the System 16 are:

$$
\alpha_{ \pm}=\frac{1}{2} \lambda_{k} \pm \frac{1}{2} \sqrt{\lambda_{k}^{2}+16 \mathrm{~K} \lambda_{k}}
$$

For a simple connected graph the spectrum $\left\{\lambda_{k}\right\}_{k=1}^{N}$ is negatively defined ${ }^{7}$ which ensures an (exponential) asymptotic convergence to the consensual state. More precisely, we have a stable focus for $\lambda_{k} \in$ ] $-16 \mathrm{~K}, 0\left[\right.$ and a stable node for $\left.\lambda_{k} \in\right]-\infty,-16 \mathrm{~K}[$. The relaxation time is given by $\tau_{\text {relax }}=\mathscr{F}^{-1}$, where $\mathscr{F}$ is the algebraic connectivity (i.e. the Fiedler number ${ }^{8}$ ) of the coupling network. Remember that $\mathscr{F}$ is the largest non-vanishing eigenvalue of the associated Laplacian matrix.

\section{Numerical simulations}

In Figures 2, 3 and 4 we report numerical simulations performed with five Hopf oscillators $(\mathrm{HO})$ defined by $H(x, y)=x^{2}+y^{2}$ and $g(H)=1-H$. Three different topologies of network interactions are considered: "All to All", "Crystal" and "All to One" (c.f.
Figure 1).

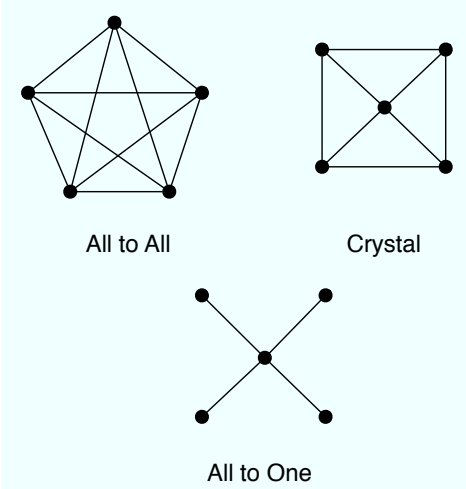

Fig. 1. Three types of network topologies with respective Fiedler number $\mathscr{F}_{(\cdot)}$ : "All to All" ( $\left.\mathscr{F}_{\mathrm{AtA}}=-5\right)$, "Crystal" $\left(\mathscr{F}_{\text {Cry }}=-3\right)$ and "All to One" $\left(\mathscr{F}_{\text {AtO }}=-1\right)$.

The learning mechanism can be observed in Figures 2,3 and 4 , and the final consensual frequency is given by Eq.(8). All three figures have the same time scale, so we can fully appreciate the fact that the convergence rates $\rho_{(\cdot)}$ clearly obey:

$$
\mathscr{F}_{\text {AtA }}<\mathscr{F}_{\text {Cry }}<\mathscr{F}_{\text {AtO }} \Rightarrow \rho_{\text {AtA }}>\rho_{\text {Cry }}>\rho_{\text {AtO }} .
$$

The smaller the Fiedler number, the faster the convergence, and thus the convergence rate explicitly depends on the topology of the network.

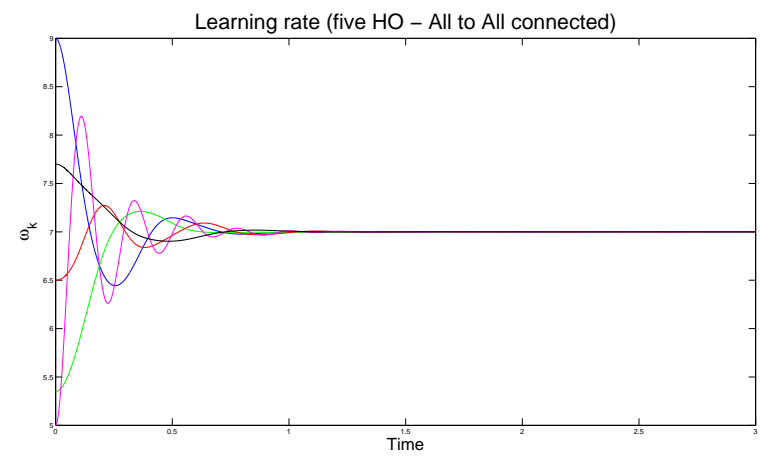

Fig. 2. Time evolution of the frequencies $\omega_{k}(t)$ for five Hopf oscillators with $\mathrm{K}_{1}=1, \mathrm{~K}_{2}=\frac{1}{2}, \mathrm{~K}_{3}=\frac{5}{4}, \mathrm{~K}_{4}=5$, $\mathrm{K}_{5}=\frac{1}{3} \omega_{1}(0)=9, \omega_{2}(0)=5.35, \omega_{3}(0)=6.5, \omega_{4}(0)=5$, $\omega_{5}(0)=7.7$ and with network topology "All to All". The consensual frequency, given by Eq.(10), is $\omega_{c}=7$. 


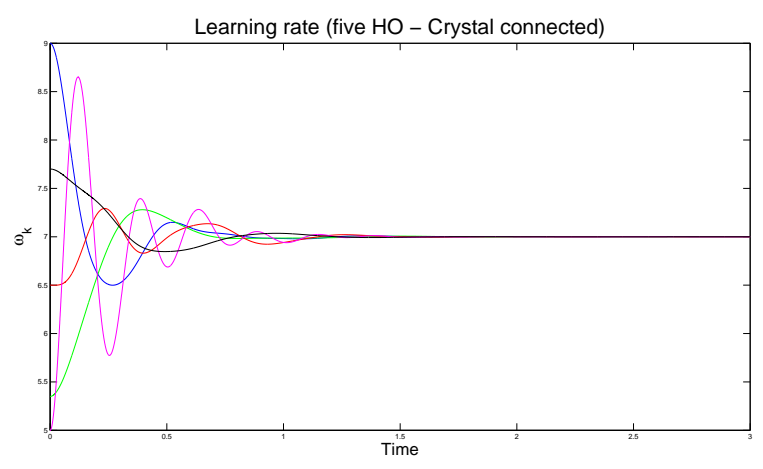

Fig. 3. Time evolution of the frequencies $\omega_{k}(t)$ for five Hopf oscillators with $\mathrm{K}_{1}=1, \mathrm{~K}_{2}=\frac{1}{2}, \mathrm{~K}_{3}=\frac{5}{4}, \mathrm{~K}_{4}=5$, $\mathrm{K}_{5}=\frac{1}{3} \omega_{1}(0)=9, \omega_{2}(0)=5.35, \omega_{3}(0)=6.5, \omega_{4}(0)=5$, $\omega_{5}(0)=7.7$ and with network topology "Crystal". The consensual frequency, given by Eq.(10), is $\omega_{c}=7$.

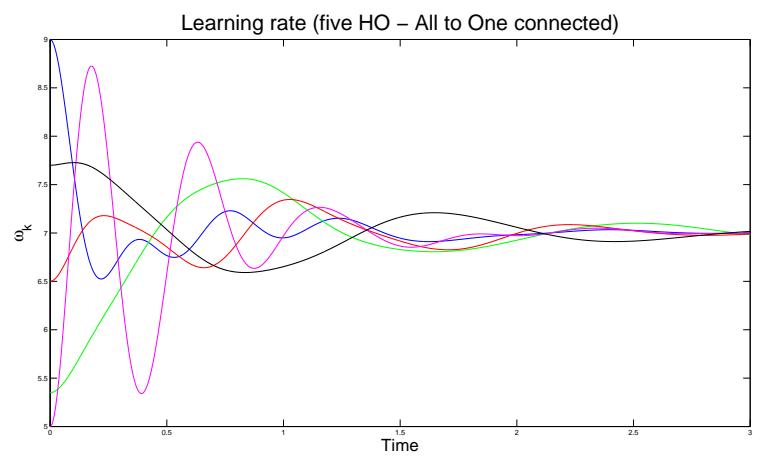

Fig. 4. Time evolution of the frequencies $\omega_{k}(t)$ for five Hopf oscillators with $\mathrm{K}_{1}=1, \mathrm{~K}_{2}=\frac{1}{2}, \mathrm{~K}_{3}=\frac{5}{4}, \mathrm{~K}_{4}=5$, $\mathrm{K}_{5}=\frac{1}{3} \omega_{1}(0)=9, \omega_{2}(0)=5.35, \omega_{3}(0)=6.5, \omega_{4}(0)=5$, $\omega_{5}(0)=7.7$ and with network topology "All to One". The consensual frequency, given by Eq.(10), is $\omega_{c}=7$.

\section{Conclusions and Perspectives}

Among the numerous possibilities of implementing the DHL learning rule, networks of limit cycle oscillators with adapting frequencies offer a yet unexplored research topics with a vast potential for practical applications. We are here able to explicitly appreciate the interplay between the DHL learning rule on one hand and the connectivity of the underlying interaction network on the other hand. In particular, the possibility to analytically calculate the consensual frequency (c.f. Eq.(8)) characterizing the circulation of the final common attractor, and the observation that the the topology of the network participate only to the convergence rate are truly remarkable features. At this preliminary stage, we do however not yet offer a complete and mathematically rigorous treatment of the rich underlying dynamics.

Several questions remain open, among them the characterization of the basin of attraction $\mathscr{B}$ of the consensual state, which could be addressed by constructing ad hoc Lyapunov functions. In particular, the dependence of $\mathscr{B}$ on the set of coupling parameters $\left\{\mathrm{K}_{k}\right\}_{k=1}^{N}$, and in the case of coupling networks which can be modeled by multi-edge graphs, remain to be studied.

\section{Acknowledgment}

The authors thank Ludovic Righetti for numerous and fruitful discussions shared during the elaboration of this work.

\section{References}

1. I.I. Blekhman, "Synchronization in science and technology," ASME Press, New York (1988).

2. L. Righetti, J. Buchli and A. Ijspeert, "Dynamic Hebbian learning in adaptive frequency oscillators," Physica D, 216, 269-281 (2006).

3. M.-O. Hongler and D.M. Ryter, "Hard Mode Stationary States Generated by Fluctuations," Zeitschrift für Physik B, 31, 333-337 (1978).

4. F. Schweitzer, W. Ebeling and B. Tilch, "Statistical mechanics of canonical-dissipative systems and applications to swarm dynamics," Physical Review E, 64, 211101-211112 (2001).

5. F. Schweitzer, "Brownian Agents and Active Particles," Berlin, Germany, Springer (2003).

6. A. Jadbabaie, N. Motee and M. Barahone, "On the Stability of the Kuramoto Model of Coupled Nonlinear Oscillators," Proc. of the American Control Conference, 5, 4296-4301 (2004).

7. J. L. Gross and J. Yellen, "Handbook of Graph Theory," Florida, U.S.A., CRC Press LLC (2004).

8. R. Olfati-Saber and R. M. Murray, "Consensual Problems in Networks of Agents With Switching Topology and Time-Delays," IEEE Trans. Automat. Contr., 49, 1520-1533 (2004). 